\title{
Design and optimization of a small horizontal axis wind turbine using BEM theory and tip loss corrections
}

\author{
Somaya Younoussi $1^{*}$, Abdeslem Ettaouil $^{1}$ \\ ${ }^{1}$ Mechanical Engineering Department, Mohammadia School of Engineers, Mohammed V university, Rabat, Morocco
}

\begin{abstract}
In this paper, an optimization approach of a small horizontal axis wind turbine based on BEM theory including De Vries and Shen et al. tip loss corrections is proposed. The optimal blade geometry was obtained by maximizing the power coefficient along the blade using the optimal angle of attack and the optimal tip speed ratio. The Newton's iterative method applied to axial induction factor was used to solve the problem. This study was conducted for a NACA4418 small wind turbine, at low wind velocity. Among the two used tip loss corrections, the De Vries correction was found to be the most suitable for this blade optimization method. The optimal design was obtained for a tip speed ratio of 5 and has recorded a power coefficient equal to 0.463 .
\end{abstract}

\section{Introduction}

Introduced by Glauert in 1935 [1], blade element momentum theory (BEM) was and still is the most used mathematical model for blade optimization. The BEM theory is a combination of two theories: The first one is the axial momentum theory and the second one is the blade element theory. The axial momentum theory was initiated by Rankine in 1865 [2] and is later improved by R.E. Froude in 1889 [3]. In this theory, the propeller is regarded as an actuator disc in which the forces are distributed continuously in the azimuth direction. Meanwhile, the blade element theory, which was developed by Drzewiecki in 1892, consists in dividing the blade on $\mathrm{N}$ elements along the radius so that each element was considered as a small airfoil. The application of this theory assumed that there is no interaction between elements and that the forces acting on an element are only due to the lift and drag applied to this element airfoil. Because of the theory's limitations, the results are generally remote from the experimental ones. In order to improve the BEM theory, many corrections were suggested. The most important correction is the tip loss correction, that was first proposed by Prandtl [4] to correct the assumption of the infinite number of blades. Furthermore, Glauert established an approximation to Prandtl's tip loss correction that can be used in BEM theory calculations, and he assumed that this correction only corrects the induced velocities not the mass flux. A refined tip loss correction was later developed by De Vries [5], to correct both the induced velocities and the mass flux. Shen et al [6] adopted the same correction of De Vries, but he proposed a new tip loss correction that predicts better the loadings in the tip region.
Another limitation of the BEM theory is that when the induction factor exceeds $a_{c}=0.5$, the theory is no longer valid. For that, Glauert [1] proposed a correction of the thrust near the tip region. In the Shen et al. [6] correction, a value of $a_{c}$ equal to $1 / 3$ was used. In the present study, the followed upper limit of induction factor is 0.4 .

In the present work, an optimization of small wind turbine blade based on BEM theory combined with De Vries and Shen et al. tip loss corrections is presented. The obtained blade geometry is then compared with the literature and the effects of the tip loss corrections on the optimal blade geometry are analyzed. The final optimal design of the blade is obtained by calculating the maximum power coefficient at different tip speed ratio.

\section{Mathematical model}

\subsection{Blade element momentum theory}

The BEM theory consists in dividing the blade into $N$ elements. For each element $d r$ of the blade, the equation of momentum and angular momentum conservation are applied. According to the second part of the BEM theory, the thrust and the torque are defined by equations (1) and (2), respectively:

$$
\begin{aligned}
& d T=0.5 B U_{r e l}^{2} C_{n} c d r \\
& d Q=0.5 B U_{r e l}^{2} C_{t} c r d r
\end{aligned}
$$

Where $\rho$ is the density of air, B is the number of blades, $\mathrm{c}$ is the chord length, $\mathrm{r}$ is the local radius, $C_{n}$ and $C_{t}$ are the normal and the tangential loads coefficients respectively, and are defined as $C_{n}=$

* Corresponding author: somayayounoussi@ research.emi.ac.ma 
$C_{L} \cos \varphi+C_{D} \sin \varphi$ and $C_{t}=C_{L} \sin \varphi-C_{D} \cos \varphi$. The relative wind velocity $U_{r e l}$ and the relative wind angle $\varphi$ are defined as follow:

$$
\begin{gathered}
\varphi=\tan ^{-1}\left(\frac{(1-a) U_{0}}{\left(1+a^{\prime}\right) \Omega r}\right) \\
U_{r e l}=\sqrt{\left[U_{0}(1-a)\right]^{2}+\left[\Omega r\left(1+a^{\prime}\right)\right]^{2}}
\end{gathered}
$$

Where $\mathrm{U}_{0}$ is the wind velocity far upstream, $\Omega$ is the rotor angular velocity, $a$ and $a^{\prime}$ are the axial and the angular induction factors respectively.

By applying the first part of BEM theory, the equations below are obtained:

$$
\begin{gathered}
d T=4 a^{\prime} \Omega^{2} r^{2}\left(1+a^{\prime}\right) \pi r d r \\
d Q=\rho U_{0} r \Omega\left[4 a^{\prime}(1-a)\right] \pi r^{2} d r
\end{gathered}
$$

By equating the two equations of thrust -(1) and (5)-, and those of torque - (2) and (6)-, the axial and tangential induction factors are then defined as:

$$
\begin{gathered}
a=\frac{1}{\frac{4(\sin \varphi)^{2}}{\sigma C_{n}}+1} \\
a^{\prime}=\frac{1}{\frac{4 \sin \varphi \cos \varphi}{\sigma C_{t}}-1}
\end{gathered}
$$
$\frac{B c}{2 \pi r}$.

With $\sigma$ is the solidity of the rotor defined by: $\sigma=$

\subsection{Prandtl tip loss correction}

In order to improve the BEM theory to be valid even for a rotor with finite number of blades, Glauert [1] presented an approximation to Prandtl's tip loss correction that can be integrated in BEM theory calculations. The proposed correction factor $F$, corrected the induced velocities. Hence the equations of thrust and torque are modified to be:

$$
\begin{gathered}
d T=\rho F U_{0}^{2} 4 a(1-a) \pi r d r \\
d Q=\rho F U_{0} \Omega\left[4 a^{\prime}(1-a)\right] \pi r^{3} d r
\end{gathered}
$$

The factor $F$ is usually expressed as:

$$
F=\frac{2}{\pi} \arccos \left(e^{\frac{-B(R-r)}{2 r \sin \varphi}}\right)
$$

$$
a=\frac{1}{\frac{4 F(\sin \varphi)^{2}}{\sigma C_{n}}+1}
$$

$$
\text { and } \quad a^{\prime}=\frac{1}{\frac{4 F \sin \varphi \cos \varphi}{\sigma C_{t}}-1}
$$

And the thrust coefficient is defined as:

$$
C_{T}=4 F a(1-a)
$$

\subsection{De Vries tip loss correction}

In addition to the induced velocity correction, De Vries [5] developed a new correction of mass flux that satisfied also the orthogonality of the induced velocity to the relative velocity. The new expressions of the induction factors are:

$$
\begin{gathered}
\frac{a F(1-a F)}{(1-a)^{2}}=\sigma \frac{C_{n}}{4(\sin \varphi)^{2}} \\
\frac{a^{\prime} F(1-a F)}{(1-a)\left(1+a^{\prime}\right)}=\sigma \frac{C_{t}}{4 \sin \varphi \cos \varphi}
\end{gathered}
$$

Using De Vries tip loss correction and Glauert high thrust correction, the equation of thrust is corrected and becomes:

$$
C_{T}= \begin{cases}4 F a(1-a F) & \text { if } \quad a \leq a_{c} \\ 4\left[a_{c}^{2} F^{2}+a F\left(1-2 F a_{c}\right)\right] & \text { if } \quad a>a_{c}\end{cases}
$$

Where $: a_{c}=1 / 3$.

\subsection{Shen et al. tip Loss Correction}

Similarly to De Vries, Shen et al.[6] corrected both the induced velocities and the mass flux. However, Shen assumed that another correction was needed for the airfoil data near the tip to take into account the threedimensional tip loss effects. Hence, a new correction factor $F 1$ was presented as a correction to the twodimensional airfoil data in the tip region. As a result, the forces coefficients expressions in the tip region are defined as:

$$
\begin{aligned}
C_{n}^{r} & =F_{1} C_{n} \\
C_{t}^{r} & =F_{1} C_{t}
\end{aligned}
$$

Where the correction factor $F 1$ is defined as:

So, the induction factors are also changed to be: 


$$
F_{1}=\frac{2}{\pi} \arccos \left(e^{-g \frac{B(R-r)}{2 r \sin \varphi}}\right)
$$

Generally, $g$ is a function of number of blades, tip speed ratio, chord distribution, pitch setting, etc. But Shen et al. proposed the following simplified form of $g$ :

$$
g=\exp [-0.125(B \lambda-21)]+0.1
$$

After correction, the induction factors become:

$$
\begin{gathered}
\frac{a F(1-a F)}{(1-a)^{2}}=\sigma \frac{F_{1} C_{n}}{4(\sin \varphi)^{2}} \\
\frac{a^{\prime} F(1-a F)}{(1-a)\left(1+a^{\prime}\right)}=\sigma \frac{F_{1} C_{t}}{4 \sin \varphi \cos \varphi}
\end{gathered}
$$
(17).

The thrust keeps the same expression of equation

\section{Proposed optimization process}

In the present work, an optimization of the wind turbine blade is done using BEM theory including De Vries and Shen et al. tip loss corrections. In these simulations, the induction factor $a$ doesn't exceed 0.4 , so that, a thrust correction is not necessary. The losses near the hub aren't of great importance as those in the tip region [7]. Therefore, only the tip region is studied. The correction factors $F$ and $F 1$ are then considered equal to 1 along the blade except the tip. In the tip region $\varphi$ tends to zero so a simplification of $F$ and $F 1$ is possible:

$$
\sin \varphi \approx \tan \varphi=\frac{1-a}{1+a^{\prime}} \frac{1}{\lambda_{r}}
$$

So, $F$ and $F 1$ become:

$$
\begin{gathered}
F=\frac{2}{\pi} \arccos \left(e^{\frac{-B(R-r)}{2 r} \frac{1+a^{\prime}}{1-a} \lambda_{r}}\right) \\
F_{1}=\frac{2}{\pi} \arccos \left(e^{-g \frac{B(R-r)}{2 r} \frac{1+a^{\prime}}{1-a} \lambda_{r}}\right)
\end{gathered}
$$

The process consists in dividing the blade into 24 elements, and maximizing the power coefficient along the blade using the optimal angle of attack and the optimal tip speed ratio. The calculation of the induction factor that provides maximum power coefficient is realized by an iterative approach using Newton method. The iterative procedure for the calculation of solution is given as follow:

(i) Initializing data.

(ii) Computing $a$ ' through the followed equation: $a^{\prime}=0.5\left[-\left(1.0+\frac{m}{\lambda_{r}}\right)+\sqrt{\left(1+\frac{m}{\lambda_{r}}\right)^{2}-\frac{4}{\lambda_{r}^{2}}\left[a^{2}-a\left(1-m \lambda_{r}\right)\right]}\right]$

where $m=\frac{C_{D}}{C_{L}}$

(iii) Computing the induction factor $a$ with the Newton method as follow:

- If $r \leq 0.8 \mathrm{R}$ :

$$
a_{n+1}=a_{n}-\frac{f\left(a_{n}\right)}{f^{\prime}\left(a_{n}\right)}
$$

Where:

$$
f\left(a_{n}\right)=\frac{d C_{p}}{d a_{n}}=\frac{d a^{\prime}}{d a_{n}}\left(1-a_{n}\right)-a^{\prime}
$$

and: $\quad f^{\prime}\left(a_{n}\right)=\frac{d^{2} a^{\prime}}{d a_{n}^{2}}\left(1-a_{n}\right)-2 \frac{d a^{\prime}}{d a_{n}} a_{n}$

- If $r>0.8 R$ :

$$
a_{n+1}=a_{n}-\frac{f\left(a_{n}\right)}{f^{\prime}\left(a_{n}\right)}
$$

Where:

$$
\begin{aligned}
f\left(a_{n}\right)= & \frac{d C_{p}}{d a_{n}}=\frac{d a^{\prime}}{d a_{n}} F\left(1-a_{n} F\right)+a^{\prime}\left(1-a_{n} F\right) \frac{d F}{d a_{n}} \\
& -a^{\prime} F\left(F+a_{n} \frac{d F}{d a_{n}}\right)
\end{aligned}
$$

and:

$$
\begin{aligned}
f^{\prime}\left(a_{n}\right)= & \frac{d^{2} C_{p}}{d a_{n}{ }^{2}}=\frac{d^{2} a^{\prime}}{d a_{n}{ }^{2}} F\left(1-a_{n} F\right)+2 \frac{d a^{\prime}}{d a_{n}} \frac{d F}{d a_{n}}\left(1-a_{n} F\right) \\
& -2 \frac{d a^{\prime}}{d a_{n}} F\left(F+a_{n} \frac{d F}{d a_{n}}\right)-2 a^{\prime}\left(F+a_{n} \frac{d F}{d a_{n}}\right) \\
& +a^{\prime} \frac{d^{2} F}{d a_{n}{ }^{2}}-2 a_{n} a^{\prime} F \frac{d^{2} F}{d a_{n}{ }^{2}}-2 a^{\prime} F \frac{d F}{d a_{n}}
\end{aligned}
$$

(iv) Verifying the convergence for the coefficient $a$. The tolerance in the present work is $10^{-5}$. If there is no convergence the step (iii) is repeated.

(v) Computing the final value of the coefficient $a$ ' through equation (27).

(vi) Computing the relative wind angle $\varphi$ through equation (3).

(vii) Computing the chord and twist angle through followed equations:

$$
\begin{gathered}
c=\frac{8 \pi r a F(1-a F)(\sin \varphi)^{2}}{B C_{n}(1-a)^{2}} \\
\theta=\varphi-\alpha_{o p t}
\end{gathered}
$$


Where $\alpha_{\text {opt }}$ is the optimal angle of attack.

(viii) Computing the thrust, and torque using equations (1) and (2).

(ix) Computing the extracted power using trapezoidal rule:

$$
\begin{gathered}
P_{\text {ext }}=\Omega\left(\frac{R-r_{\text {hub }}}{N-1}\right)\left[\left(\frac{G(1)+G(N-1)}{2}\right)+\sum_{2}^{N-2} G(i)\right] \\
\text { with } \quad G(i)=0.5 B \operatorname{pr}(i) U_{r e l}(i)^{2} C_{t}(i) c(i)
\end{gathered}
$$

\section{(x) Computing the power coefficient.}

\section{Results and discussion}

The calculation of the solution is programmed using FORTRAN 95 code. The obtained results are compared with the optimized design of [8] obtained by using the optimum rotor theory. The characteristics of the rotor wind turbine used in this work are the same used by [8] and are given in the table1.

Table1. The design characteristics of the used wind turbine blade.

\begin{tabular}{|l|l|}
\multicolumn{2}{|c|}{ blade. } \\
\hline Profile type & NACA4418 \\
\hline Optimal Angle of attack & $6.5^{\circ}$ \\
\hline Lift coefficient & 1.209 \\
\hline Lift to drag coefficient & 44.447 \\
\hline Radius & $0.65 \mathrm{~m}$ \\
\hline Hub radius & $0.05 \mathrm{~m}$ \\
\hline Number of blades & 3 \\
\hline Design wind speed & $8 \mathrm{~m} / \mathrm{s}$ \\
\hline Optimal Design tip speed ratio & 4 \\
\hline
\end{tabular}

The distributions of the chord and the twist angle obtained using this program are given in the Fig.1. Along the blade other than the tip, the chord and the twist angle have approximately the same distribution as the model of [8]. But in the tip region, the design obtained by the present work using Shen et al. shows an augmentation of chord near the tip, while with the use of De Vries the chord decreases in the tip region. Even a slight decrease of twist angle is observed in this region for both the two tip loss corrections.

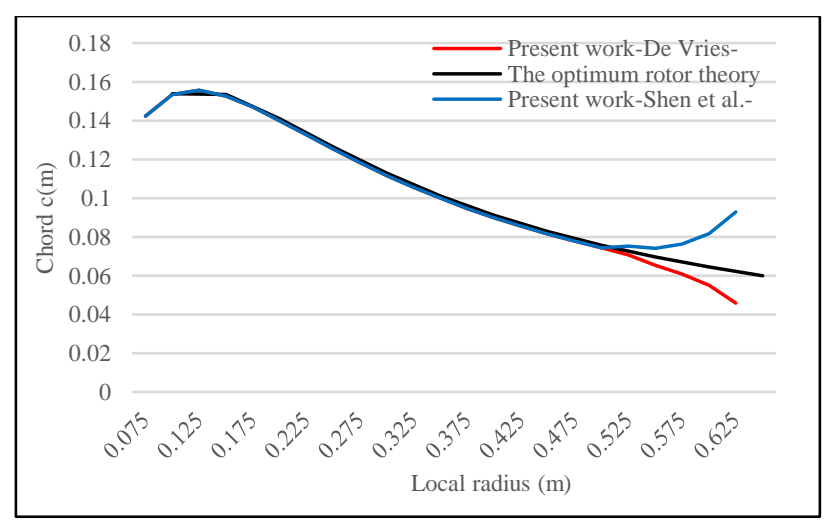

a: Chord

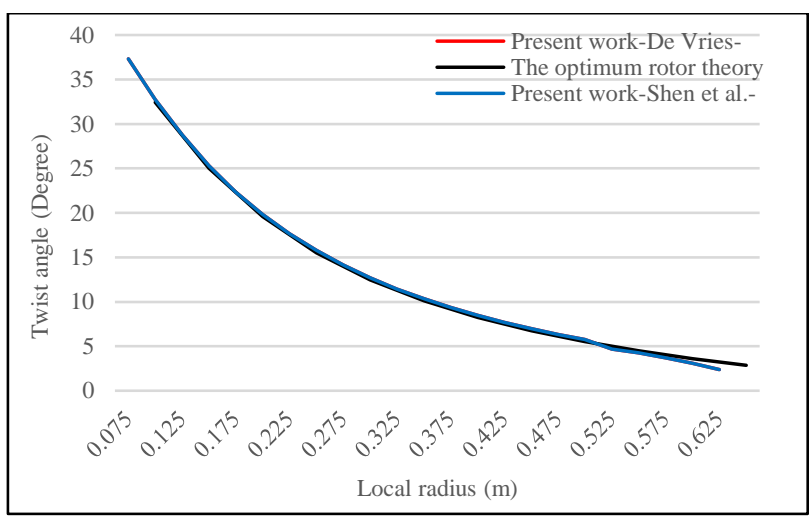

b: Twist angle

Fig.1. Optimal Chord (a) and Twist (b) distributions using De Vries and Shen et al. tip loss corrections at $\lambda=4$.

The tip correction of Shen et al. gives an anticipated result. The design obtained is questionable from an aerodynamic point of view. In [7], this problem is due to the fact that in the expression of the correction factor $F 1$, $g$ must be a function of $B, \lambda$ and chord distribution. But, with a lack of data $g$ is simplified and is given in terms of $B$ and $\lambda$ only. So, the $F 1$ doesn't depend on $c$, and as $\mathrm{F} 1$ decreases in the tip region the chord increases. As a result, De Vries tip loss correction is selected to be used in the present blade optimization approach.

Fig.2. presents the distributions of torque and thrust forces of the optimum rotor resulted from both the present work using De Vries tip loss correction and the optimum rotor theory. Near the hub the torque tends to 0 , that means that the power produced in this region is not important as it was assumed previously. Except the tip region, for the two models, the torque starts to increase slowly, and the thrust increases linearly. But, in the tip region, while the forces of the optimum rotor keep increasing, those of the present work reach a maximum value and then curve due to the correction effects. The decrease of loads near the tip in the present model is due to the tip vortex that reduce the lifting capability. Along the blade except the tip region the proposed model presents better results for thrust and torque compared to those of the optimum rotor theory model. In the tip region the problem of the over-predicted forces given by the optimum rotor theory is solved by the present work.

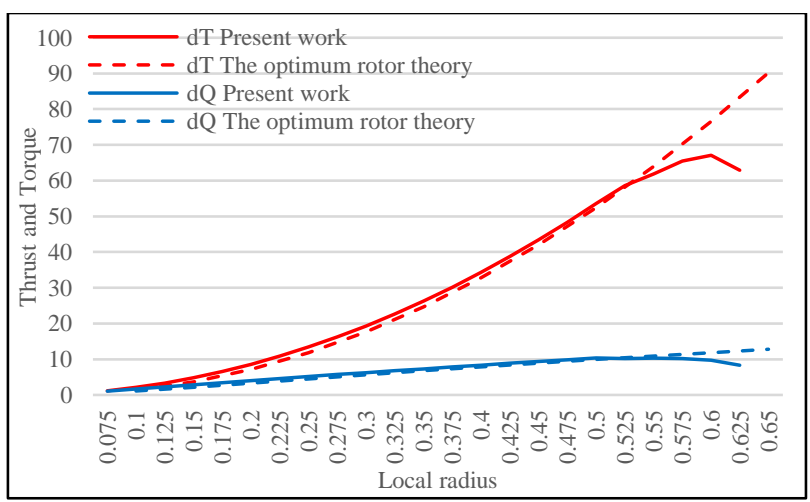

Fig.2. The torque dQ and the thrust dT distributions of the optimum rotor using De Vries correction. 
The power coefficient obtained by the optimum rotor theory is equal to 0.465 , but after correction a coefficient of 0.461 is obtained. So, the inclusion of De Vries tip loss correction in the optimization process decreases $C p$ by $0.4 \%$.

The design tip speed ratio is varied thereafter in order to get maximum power. The proposed program work for a range of $\lambda \geq 2$. Fig.3, shows the variation of power coefficient with tip speed ratio. The maximum power coefficient of 0.463 is obtained at $\lambda=5$.

Finally, the new model obtained after optimization has the chord and twist distribution illustrated in Fig.4.

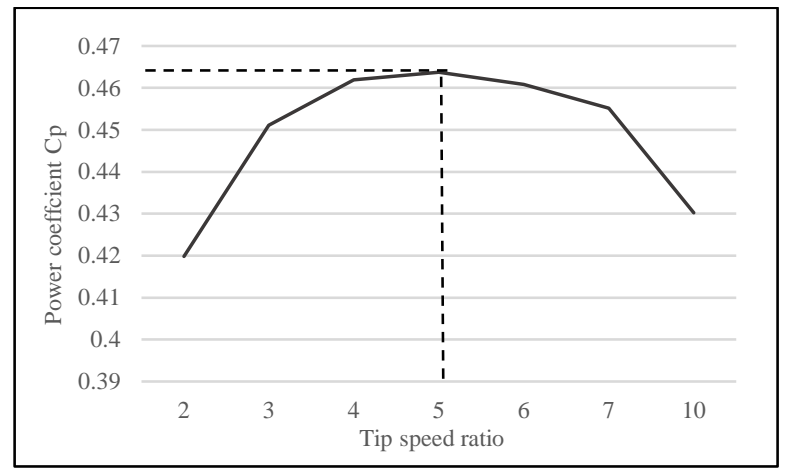

Fig.3. Variation of power coefficient with design tip speed ratio $\lambda=5$.

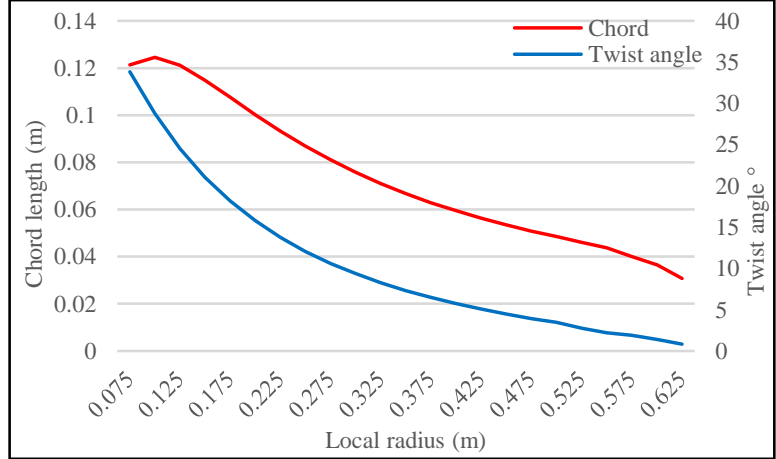

Fig.4. Optimal Chord and Twist distributions obtained at at a wind speed of $8 \mathrm{~m} / \mathrm{s}$.

\section{Conclusion}

The proposed approach for the wind turbine blade optimization was applied for NACA4418 small wind turbine. The De Vries tip loss correction is found to be the most appropriate one for the present blade optimization approach. The inclusion of tip loss correction decreases the power coefficient by $0.4 \%$. The maximum power extraction of 0.463 is recorded at tip speed ratio of 5 . In the future work, a CFD study of the obtained optimal design will be done in order to validate the paper's results.

\section{References}

1. H. Glauert, in Aerodynamic Theory: A General Review of Progress Under a Grant of the Guggenheim Fund for the Promotion of Aeronautics, edited by W. F. Durand (Springer, Berlin, Heidelberg, 1935), pp. 169-360

2. W. J. M. RANKINE, Transactions of the Institution of Naval Architects 6, (1865)

3. R. E. FROUDE, Trans. Inst. Naval Architects 30, 390 (1889)

4. L. Prandtl and A. Betz, Vier Abhandlungen zur Hydrodynamik und Aerodynamik (Universitätsverlag Göttingen, 2010)

5. O. de Vries, Fluid Dynamic Aspects of Wind Energy Conversion (ADVISORY GROUP FOR AEROSPACE RESEARCH AND DEVELOPMENT NEUILLY-SUR-SEINE (FRANCE), 1979)

6. W. Z. Shen, R. Mikkelsen, J. N. Sørensen, and C. Bak, Wind Energy 8, 457 (2005)

7. M. J. Clifton-Smith, Wind Engineering 33, 477 (2009)

8. M. A. Abdelrahman, O. E. Abdellatif, M. Moawed, A. Eliwa, and S. Misak, in 2015 16th International Scientific Conference on Electric Power Engineering (EPE) (IEEE, Kouty Nad Desnou, Czech Republic, 2015), pp. 754-759

9. Y. El khchine and M. Sriti, Energy Procedia 118, (2017) 